\title{
Carotico-Cavernous Fistula Presenting with Proptosis of Contralateral Eye: A Case Report
}

\author{
Subedi K ${ }^{1}$, Chataut $D^{1}$, Khatiwada $P^{2}$, Ghimire RK ${ }^{1}$, Ansari MA ${ }^{1}$ \\ ${ }^{1}$ Department of Radiology and Imaging, Institute of Medicine, Tribhuvan University \\ Teaching Hospital, Kathmandu, Nepal, ${ }^{2}$ Kanti Children Hospital, Kathmandu, Nepal
}

\begin{abstract}
Spontaneous carotico-cavernous fistula occurring post partum is a rare entity. We report a rare case of post partum spontaneous carotico-cavernous fistula with paradoxical proptosis of contralateral eye confirmed by computed tomography and cerebral angiography.
\end{abstract}

Keywords: Carotico-cavernous fistula, cerebral angiography, Computed tomography

\section{Introduction}

Carotid-cavernous fistula (CCF) is an abnormal communication at the base of the skull between the carotid artery and the cavernous sinus. ${ }^{1}$ Though trauma has been reported as the major cause, few cases of spontaneous CCF occurring immediately in the post partum period have been reported. ${ }^{2,3}$ Bilateral orbital findings can be present due to the transmission of increased venous pressure to the contralateral cavernous sinus via the circular sinus. ${ }^{4}$ Paradoxical involvement of the contralateral cavernous sinus has been reported following trauma.$^{5}$

We report a rare case of spontaneous post partum $\mathrm{CCF}$ with proptosis of contralateral eye confirmed angiographically.

\section{Case report}

A 30years old female presented with rapidly

Correspondence to:Dr. Kamal Subedi,
Department of Radiology and Imaging, Institute of Medicine, Tribhuvan University Teaching Hospital, Kathmandu, Nepal E-mail: ksubedi80@gmail.com progressive pulsatile proptosis of left eye (Fig a). Her problem started spontaneously on $7^{\text {th }}$ post partum day with diminution of vision, swelling and ptosis of right eye which subsequently subsided to involve the left eye few days later. There was no history of trauma. On examination, there was ptosis, pulsatile proptosis, conjunctival chemosis, swollen lids and complete ophthalmoplegia in the left eye. Visual acquity was $6 / 24$ in the left eye and the vision was normal in the right eye at the time of presentation. Bruit was positive over the left eye. Systemic examination was within normal limits.

Contrast enhanced CT (CECT) of the orbit and head were obtained followed by cerebral angiogram.

CECT of orbit revealed gross axial proptosis of the left eye with thickening of all the extraocular muscles as well as the optic nerve sheath complex. Preseptal thickening was also noted. Right orbital contents in both intra and extra-conal compartments were normal. The extraocular muscles and the optic nerve sheath complex were normal in right side (Fig b, c). 


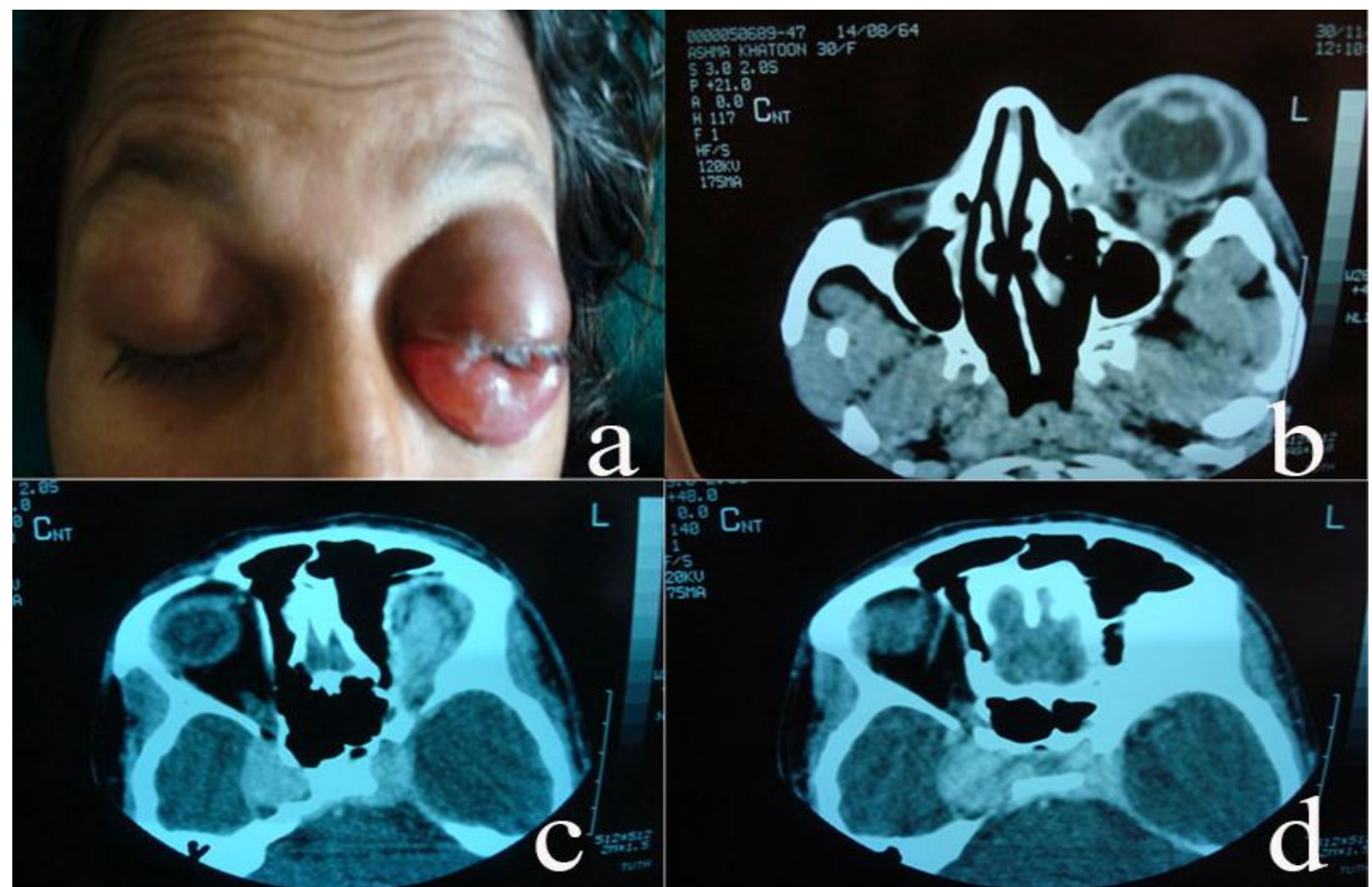

Fig a: Photograph of the patient showing gross proptosis of left eye. Fig b to d: CECT orbit and brain showing left proptosis, normal right globe and marked enlargement and enhancement of the right cavernous sinus.

CECT of brain revealed marked enlargement and enhancement of the right cavernous sinus (Fig c,d). CECT features were suggestive of right carotico-cavernous fistula. Patient was subjected to conventional cerebral angiogram to explain paradoxical symptoms.

Cerebral angiogram (right internal carotid arteriogram) images showed well defined lobulated contrast filled structure in the cavernous segment of right internal carotid artery. Contrast was also noted in the contralateral cavernous sinus in the arterial phase indicating fistulous communication of the right ICA with right cavernous sinus and intercavernous passage of contrast via circular sinus. Angiogram also showed early filling of cavernous sinus, inferior petrosal sinus and internal jugular vein in the left side (Fig e,f).

\section{Discussion}

Caroticocavernous have been anatomically and angiographically classified by Barrow et al in 1985. They can be classified according to three criteria: 1) pathogenetically into spontaneous or traumatic fistulas; 2)hemodynamically into high-flow or lowflow fistulas; and 3) angiographically into direct or dural fistulas. ${ }^{1}$

Direct carotico-cavernous fistula result from direct communication between the internal carotid artery and the cavernous sinus whereas indirect fistula is supplied by dural branches of the external carotid artery but can also be supplied by dural branches of the internal carotid artery. Pregnancy has been implicated as cause of indirect fistula. ${ }^{1,2,3}$

Our patient had a high flow indirect fistula as no direct extravasation of contrast was 


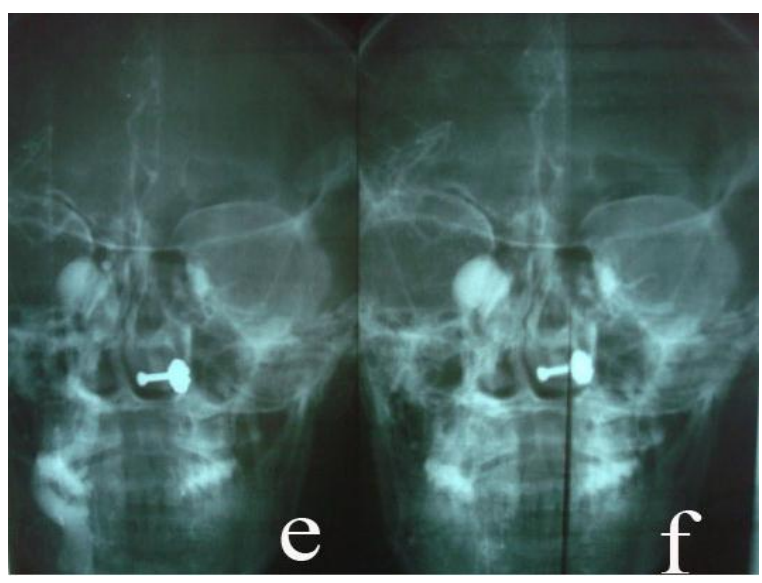

Fig e \& f: Cerebral angiogram (patient's right is towards observer's left): Well defined lobulated contrast filled structure in the cavernous segment of right internal carotid artery. Contrast is also noted in the contralateral cavernous sinus in the arterial phase indicating fistulous communication of the right ICA with right cavernous sinus and intercavernous passage of contrast via circular sinus. Early filling of cavernous sinus, inferior petrosal sinus and internal jugular vein is also noted in the left side.

noted from the right internal carotid artery in the angiograms. Several authors have reported the association of caroticocavernous fistula with pregnancy following a normal delivery and an abortion. ${ }^{6,7,8}$ The exact etiology remains unknown but authors have implicated the hemodynamic stress of pregnancy with resultant hypertension ${ }^{8}$ as well as the hormone relaxin.,

The disappearance of ipsilateralproptosis followed by appearance in the contralateral eye remains elusive in our case. Partial thrombosis of the ipsilateral cavernous sinus and decompression through the circular sinus probably explain the findings. However, no clinicoradiological finding were suggestive of ipsilateral cavernous sinus thrombosis.

\section{Conclusion}

To conclude, spontaneous caroticocavernous fistula following pregnancy is a rare entity of unknown etiology. Paradoxical symptoms as in our case are even rarer. It should be diagnosed early and treatment should be planned early to prevent the complications like life threatening bleeding.

\section{References}

1. Barrow DL, Spector RH, Braun IF, Landman JA, Tindall SC, Tindall GT. Classification and treatment of spontaneous carotid-cavernous sinus fistulas. J Neurosurg 1985;62:248-256.

2. Toya S, Shiobara R, Izumi J, Shinomiya Y, Shiga H, Kimura C. Spontaneous carotid-cavernous fistula during pregnancy or in the post partum stage. $\mathbf{J}$ Neurosurg 1981;54: 252-256.

3. Prabhakar $\mathrm{H}$, Bithal PK, Jagia $M$. Spontaneous Carotico-Cavernous Fistula Following Normal Labor. The Internet Journal of Anesthesiology2004. Volume 8 Number 2.

4. Sutton D. Carotid-cavernous fistula. In: Sutton D, editor. Text Book of Radiology and Imaging. Edinburg: Churchill Livingstone; 2003:1584.

5. Hawkins TD. Traumatic carotidcavernous fistula with contralateral proptosis.Clinical Radiology 1986;37(5):509-512.

6. Newton $\mathrm{T} H$, Hoyt $\mathrm{W}$ F. Dural arteriovenous shunts in the region of the cavernous sinus. Neuroradiology 1970;1:71-81.

7. Taniguchi R M, Goree J A, Odom G L. Spontaneous carotid-cavernous shunts presenting diagnostic problems. J Neurosurg 1971;35:384-391.

8. Walker A E, Allegre G. Carotidcavernous fistulas. Surgery 1056;39:411422.

9. Lin $\mathrm{T} \mathrm{K}$, Chang $\mathrm{C} \mathrm{N}$, Wai $\mathrm{Y} \mathrm{Y}$. Spontaneous intracerebral hematoma from occult carotid-cavernous fistula during pregnancy and puerperium. J Neurosurg1992;76:714-717. 